\title{
ON ASYMPTOTIC EXPANSIONS OF GENERALIZED BERGMAN KERNELS ON SYMPLECTIC MANIFOLDS
}

\author{
YURI A. KORDYUKOV \\ Dedicated to the 130th anniversary of Vladimir Ivanovich Smirnov's birth
}

\begin{abstract}
A full off-diagonal asymptotic expansion is established for the generalized Bergman kernels of the renormalized Bochner Laplacians associated with high tensor powers of a positive line bundle over a compact symplectic manifold. As an application, the algebra of Toeplitz operators on the symplectic manifold associated with the renormalized Bochner Laplacian is constructed.
\end{abstract}

\section{INTRODUCTION}

In this paper, we study the asymptotic behavior of the generalized Bergman kernels of the renormalized Bochner-Laplacians associated to high tensor powers of a positive line bundle over a compact symplectic manifold. So we consider a compact symplectic manifold $(X, \omega)$ of dimension $2 n$. Let $\left(L, h^{L}\right)$ be a Hermitian line bundle on $X$ with a Hermitian connection $\nabla^{L}: C^{\infty}(X, L) \rightarrow C^{\infty}\left(X, T^{*} X \otimes L\right)$. The curvature of this connection is given by $R^{L}=\left(\nabla^{L}\right)^{2}$. We will assume that $L$ satisfies the prequantization condition:

$$
\frac{i}{2 \pi} R^{L}=\omega
$$

Thus, $[\omega] \in H^{2}(X, \mathbb{Z})$. Let $\left(E, h^{E}\right)$ be a Hermitian vector bundle on $X$ with Hermitian connection $\nabla^{E}$, and let $R^{E}$ be the curvature of $\nabla^{E}$.

Let $g$ be a Riemannian metric on $X$. Let $J_{0}: T X \rightarrow T X$ be a skewsymmetric operator such that

$$
\omega(u, v)=g\left(J_{0} u, v\right), \quad u, v \in T X .
$$

Consider the operator $J: T X \rightarrow T X$ given by $J=J_{0}\left(-J_{0}^{2}\right)^{-1 / 2}$. Then $J$ is an almost complex structure compatible with $\omega$ and $g$, that is, $g(J u, J v)=$ $g(u, v), \omega(J u, J v)=\omega(u, v)$ and $\omega(u, J u) \geq 0$ for any $u, v \in T X$.

Let $\nabla^{T X}$ be the Levi-Chivita connection of the metric $g$. For any $p \in \mathbb{N}$ denote by $L^{p}$ the $p$ th tensor power of $L$. Let $\nabla^{L^{p} \otimes E}: C^{\infty}\left(X, L^{p} \otimes E\right) \rightarrow$

2000 Mathematics Subject Classification. Primary 58J37; Secondary 53D50.

Key words and phrases. Symplectic manifold, Bochner Laplacian, Bergman kernel, asymptotics, Toeplitz operators, quantization.

The research is supported by the grant of Russian Science Foundation (project no. 17-11-01004). 
$C^{\infty}\left(X, L^{p} \otimes E \otimes T^{*} X\right)$ be the connection on $L^{p} \otimes E$ induced by $\nabla^{L}$ and $\nabla^{E}$. Denote by $\Delta^{L^{p} \otimes E}$ the induced Bochner-Laplacian acting on $C^{\infty}\left(X, L^{p} \otimes E\right)$ by the formula

$$
\Delta^{L^{p} \otimes E}=\left(\nabla^{L^{p} \otimes E}\right)^{*} \nabla^{L^{p} \otimes E},
$$

where $\left(\nabla^{L^{p} \otimes E}\right)^{*}: C^{\infty}\left(X, L^{p} \otimes E \otimes T^{*} X\right) \rightarrow C^{\infty}\left(X, L^{p} \otimes E\right)$ stands for the formal adjoint of the operator $\nabla^{L^{p} \otimes E}$, and by $\Delta_{p}$ the renormalized Bochner Laplacian acting on $C^{\infty}\left(X, L^{p} \otimes E\right)$ by the formula

$$
\Delta_{p}=\Delta^{L^{p} \otimes E}-p \tau,
$$

where $\tau \in C^{\infty}(X)$ is given by

$$
\tau(x)=-\pi \operatorname{Tr}\left[J_{0}(x) J(x)\right], \quad x \in X .
$$

The renormalized Bochner-Laplacian $\Delta_{p}$ was introduced by V. Guillemin and A. Uribe in [7]. When $(X, \omega)$ is a Kaehler manifold, it is twice the corresponding Kodaira-Laplacian on functions $\square^{L^{p}}=\bar{\partial}^{L^{p} *} \bar{\partial}^{L^{p}}$. The asymptotics of its spectrum as $p \rightarrow \infty$ was studied in 4, 5, 7, 12, 14.

Denote by $\sigma\left(\Delta_{p}\right)$ the spectrum of $\Delta_{p}$ in $L^{2}\left(X, L^{p} \otimes E\right)$. By [14, Corollary 1.2] (see also [3, 4, 5, 7]), there exists a constant $C_{L}>0$ such that

$$
\sigma\left(\Delta_{p}\right) \subset\left[-C_{L}, C_{L}\right] \cup\left[2 p \mu_{0}-C_{L},+\infty\right),
$$

for any $p$, where $\mu_{0}>0$ is given by

$$
\mu_{0}=\inf _{u \in T_{x} X, x \in X} \frac{i R_{x}^{L}(u, J(x) u)}{|u|_{g}^{2}} .
$$

Consider the linear subspace $\mathcal{H}_{p} \subset L^{2}\left(X, L^{p} \otimes E\right)$ spanned by the eigensections of $\Delta_{p}$ corresponding to eigenvalues in $\left[-C_{L}, C_{L}\right]$. Let $P_{\mathcal{H}_{p}}$ be the orthogonal projection in $L^{2}\left(X, L^{p} \otimes E\right)$ onto $\mathcal{H}_{p}$. The smooth kernel $P_{q, p}\left(x, x^{\prime}\right)$, $x, x^{\prime} \in X$, of the operator $\left(\Delta_{p}\right)^{q} P_{\mathcal{H}_{p}}$ with respect to the Riemannian volume form $d v_{X}$ is called a generalized Bergman kernel of $\Delta_{p}$.

We are interested in the asymptotic behavior of the generalized Bergman kernel $P_{q, p}\left(x, x^{\prime}\right)$ as $p \rightarrow \infty$. First, we recall that, by [14], for any $m \in \mathbb{N}$ and $\varepsilon>0$, we have

$$
\left|P_{q, p}\left(x, x^{\prime}\right)\right|_{C^{m}}=\mathcal{O}\left(p^{-\infty}\right)
$$

if $d\left(x, x^{\prime}\right)>\varepsilon$. To describe the asymptotic expansion of $P_{q, p}\left(x, x^{\prime}\right)$ near the diagonal, we introduce normal coordinates near an arbitrary point $x_{0} \in X$.

Let $a^{X}$ be the injectivity radius of the Riemannian manifold $(X, g)$. We denote by $B^{X}\left(x_{0}, r\right)$ and $B^{T_{x_{0}} X}(0, r)$ the open balls in $X$ and $T_{x_{0}} X$ with center $x_{0}$ and radius $r$, respectively. We identify $B^{T_{x_{0}} X}\left(0, a^{X}\right)$ with $B^{X}\left(x_{0}, a^{X}\right)$ via the exponential map $\exp _{x_{0}}^{X}$. Furthermore, we choose trivializations of the bundles $L$ and $E$ over $B^{X}\left(x_{0}, a^{X}\right)$, identifying their fibers $L_{Z}$ and $E_{Z}$ at $Z \in B^{T_{x_{0}} X}\left(0, a^{X}\right) \cong B^{X}\left(x_{0}, a^{X}\right)$ with the spaces $L_{x_{0}}$ and $E_{x_{0}}$ by parallel transport with respect to the connections $\nabla^{L}$ and $\nabla^{E}$ along the curve $\gamma_{Z}:[0,1] \ni u \rightarrow \exp _{x_{0}}^{X}(u Z)$. Denote by $\nabla^{L^{p} \otimes E}$ and $h^{L^{p} \otimes E}$ the connection and the Hermitian metric on the trivial bundle with fiber $\left(L^{p} \otimes E\right)_{x_{0}}$ induced by these trivializations. 
Let $d v_{T X}$ denote the Riemannian volume form of the Euclidean space $\left(T_{x_{0}} X, g_{x_{0}}\right)$. We define a smooth function $\kappa$ on

$$
B^{T_{x_{0}} X}\left(0, a^{X}\right) \cong B^{X}\left(x_{0}, a^{X}\right)
$$

by the equation

$$
d v_{X}(Z)=\kappa(Z) d v_{T X}(Z), \quad Z \in B^{T_{x_{0}} X}\left(0, a^{X}\right) .
$$

The almost complex structure $J_{x_{0}}$ induces a decomposition

$$
T_{x_{0}} X \otimes_{\mathbb{R}} \mathbb{C}=T_{x_{0}}^{(1,0)} X \oplus T_{x_{0}}^{(0,1)} X
$$

where $T_{x_{0}}^{(1,0)} X$ and $T_{x_{0}}^{(0,1)} X$ are the eigenspaces of $J_{x_{0}}$ corresponding to eigenvalues $i$ and $-i$ respectively. Denote by $\operatorname{det}_{\mathbb{C}}$ the determinant function of the complex space $T_{x_{0}}^{(1,0)} X$. Put

$$
\mathcal{J}_{x_{0}}=-2 \pi i J_{0}
$$

Then $\mathcal{J}_{x_{0}}: T_{x_{0}}^{(1,0)} X \rightarrow T_{x_{0}}^{(1,0)} X$ is positive, and $\mathcal{J}_{x_{0}}: T_{x_{0}} X \rightarrow T_{x_{0}} X$ is skew-symmetric. We define a function $\mathcal{P}=\mathcal{P}_{x_{0}} \in C^{\infty}\left(T_{x_{0}} X \times T_{x_{0}} X\right)$ by

$$
\begin{aligned}
& \mathcal{P}\left(Z, Z^{\prime}\right) \\
& \quad=\frac{\operatorname{det}_{\mathbb{C}} \mathcal{J}_{x_{0}}}{(2 \pi)^{n}} \exp \left(-\frac{1}{4}\left\langle\left(\mathcal{J}_{x_{0}}^{2}\right)^{1 / 2}\left(Z-Z^{\prime}\right),\left(Z-Z^{\prime}\right)\right\rangle+\frac{1}{2}\left\langle\mathcal{J}_{x_{0}} Z, Z^{\prime}\right\rangle\right) .
\end{aligned}
$$

It is the Bergman kernel of the second order differential operator $\mathcal{L}_{0}$ on $C^{\infty}\left(T_{x_{0}} X, E_{x_{0}}\right)$ given by

$$
\mathcal{L}_{0}=-\sum_{j=1}^{2 n}\left(\nabla_{e_{j}}+\frac{1}{2} R_{x_{0}}^{L}\left(Z, e_{j}\right)\right)^{2}-\tau\left(x_{0}\right),
$$

where $\left\{e_{j}\right\}_{j=1, \ldots, 2 n}$ is an orthonormal base in $T_{x_{0}} X$. Here, for $U \in T_{x_{0}} X$, we denote by $\nabla_{U}$ the ordinary operator of differentiation in the direction $U$ on the space $C^{\infty}\left(T_{x_{0}} X, E_{x_{0}}\right)$. Thus, $\mathcal{P}$ is the smooth kernel (with respect to $\left.d v_{T X}\right)$ of the orthogonal projection in $L^{2}\left(T_{x_{0}} X, E_{x_{0}}\right)$ to the kernel $N$ of $\mathcal{L}_{0}$.

Consider the fiberwise product

$$
T X \times_{X} T X=\left\{\left(Z, Z^{\prime}\right) \in T_{x_{0}} X \times T_{x_{0}} X: x_{0} \in X\right\} .
$$

Let $\pi: T X \times_{X} T X \rightarrow X$ be the natural projection given by $\pi\left(Z, Z^{\prime}\right)=x_{0}$. The kernel $P_{q, p}\left(x, x^{\prime}\right)$ induces a smooth section $P_{q, p, x_{0}}\left(Z, Z^{\prime}\right)$ of the vector bundle $\pi^{*}(\operatorname{End}(E))$ on $T X \times_{X} T X$ defined for all $x_{0} \in X$ and $Z, Z^{\prime} \in T_{x_{0}} X$ with $|Z|,\left|Z^{\prime}\right|<a_{X}$.

The main result of the paper is the following theorem, which states the existence of the full off-diagonal asymptotic expansion of the generalized Bergman kernel $P_{q, p}$ as $p \rightarrow \infty$.

Theorem 1. There exists $\varepsilon \in\left(0, a^{X}\right)$ such that, for any $j, m, m^{\prime} \in \mathbb{N}$, $j \geq 2 q$, there exist positive constants $C, c$ and $M$ such that for any $p \geq 1$, $x_{0} \in X$ and $Z, Z^{\prime} \in T_{x_{0}} X,|Z|,\left|Z^{\prime}\right|<\varepsilon$, we have 


$$
\begin{aligned}
& \sup _{|\alpha|+\left|\alpha^{\prime}\right| \leq m} \mid \frac{\partial^{|\alpha|+\left|\alpha^{\prime}\right|}}{\partial Z^{\alpha} \partial Z^{\prime \alpha^{\prime}}}\left(\frac{1}{p^{n}} P_{q, p, x_{0}}\left(Z, Z^{\prime}\right)\right. \\
& \left.\quad-\sum_{r=2 q}^{j} F_{q, r, x_{0}}\left(\sqrt{p} Z, \sqrt{p} Z^{\prime}\right) \kappa^{-\frac{1}{2}}(Z) \kappa^{-\frac{1}{2}}\left(Z^{\prime}\right) p^{-\frac{r}{2}+q}\right)\left.\right|_{\mathcal{C}^{m^{\prime}}(X)} \\
& \leq C p^{-\frac{j-m+1}{2}+q}\left(1+\sqrt{p}|Z|+\sqrt{p}\left|Z^{\prime}\right|\right)^{M} \exp \left(-c \sqrt{\mu_{0} p}\left|Z-Z^{\prime}\right|\right)+\mathcal{O}\left(p^{-\infty}\right),
\end{aligned}
$$

where

$$
F_{q, r, x_{0}}\left(Z, Z^{\prime}\right)=J_{q, r, x_{0}}\left(Z, Z^{\prime}\right) \mathcal{P}_{x_{0}}\left(Z, Z^{\prime}\right),
$$

the $J_{q, r, x_{0}}\left(Z, Z^{\prime}\right)$ are polynomials in $Z, Z^{\prime}$, depending smoothly on $x_{0}$, with the same parity as $r$ and $\operatorname{deg} J_{q, r, x_{0}} \leq 3 r$.

Here $\mathcal{C}^{m^{\prime}}(X)$ is the $\mathcal{C}^{m^{\prime}}$-norm for the parameter $x_{0} \in X$. We say that $G_{p}=\mathcal{O}\left(p^{-\infty}\right)$ if for any $l, l_{1} \in \mathbb{N}$, there exists $C_{l, l_{1}}>0$ such that $\mathcal{C}^{l_{1}}$-norm of $G_{p}$ is estimated from above by $C_{l, l_{1}} p^{-l}$.

The full off-diagonal asymptotic expansion of the Bergman kernel of the $\operatorname{spin}^{c}$ Dirac operator associated to a positive line bundle on a compact symplectic manifold was proved by X. Dai, K. Liu and X. Ma [ 6 , Theorem 4.18'] (see also [13, Theorem 4.2.1]). Their approach is inspired by the local index theory, especially by the analytic techniques of Bismut and Lebeau. In that case, it is very important that the eigenvalues of the associated Laplacian are either 0 or tend to $+\infty$.

In the current situation, the renormalized Bochner-Laplacian possibly have different bounded eigenvalues. Nevertheless, in [14, X. Ma and G. Marinescu developed the method to obtain a weaker result, a near diagonal asymptotic expansion of the generalized Bergman kernels of the renormalized Bochner Laplacian [14, Theorem 1.19] (see also [13, Theorem 4.1.24]), which turned out to be sufficient for many applications. The paper [14] also contains computations of some coefficients $F_{q, r, x_{0}}$.

In this paper, we modify the technique of Ma and Marinescu to prove the full off-diagonal asymptotic expansion of the generalized Bergman kernels of the renormalized Bochner Laplacian. We follow the strategy of [6, 14]. So the first step is localization of the problem in a neighborhood of the diagonal. Then we rescale the operator in the normal coordinates introduced above and obtain its formal asymptotic expansion as $p \rightarrow \infty$. Finally, we use the Riesz formula, the formal power series technique, Sobolev norm estimates and Sobolev embedding theorems. The most essential improvement which allows us to extend the domain of validity of asymptotic expansions is the use of weighted Sobolev spaces and weighted estimates. Here we apply the technique that was used earlier in [9, 10, 1, 2] to prove pointwise estimates for the kernels of functions of elliptic differential operators on noncompact manifolds. 
As an immediate application of Theorem 1, we construct the algebra of Toeplitz operators on the symplectic manifold $X$ associated with the renormalized Bochner Laplacian. Actually, once we prove the full off-diagonal asymptotic expansion of the generalized Bergman kernels, we can easily get a characterization of Toeplitz operators and prove that these operators form an algebra, following the arguments of [15. In the process of preparation of this paper, X. Ma and G. Marinescu informed me about the preprint [8], where they constructed the algebra of Toeplitz operators associated with the renormalized Bochner Laplacian, by using asymptotic expansions of the generalized Bergman kernels in two complimentary domains covering the manifold [8, (2.5) and Theorem 2.1] (see also [11]). These expansions are stronger than the near diagonal expansions proved in [14], but weaker than the full off-diagonal ones established in the present paper.

The paper is organized as follows. In $\$ 2$ we recall the results of [14, Sections 1.1 and 1.2] on the localization and the rescaling of the problem which allow us to obtain a formal asymptotic expansion of the renormalized Bochner-Laplacian as $p \rightarrow \infty$. In $\$ 3$, we derive the weighted norm estimates for the resolvent of the renormalized Bochner-Laplacian $\mathcal{L}_{t}$. In $\$ 4$, we derive the weighted norm estimates for the generalized Bergman projection $\mathcal{P}_{q, t}$ associated with the operator $\mathcal{L}_{t}$ and its derivatives of an arbitrary order with respect to $t$. In $\$$, we first use the estimates of Section 4 to derive the weighted norm estimates for the remainders in the asymptotic formula for the generalized Bergman projection $\mathcal{P}_{q, t}$. Then Sobolev embedding theorem allows us to obtain pointwise estimates of the remainders in the asymptotic formula for the generalized Bergman kernels. Finally, writing these pointwise estimates in the initial coordinates, we complete the proof of the main theorem. $\sqrt{6}$ is devoted to Toeplitz operators.

The author is grateful to X. Ma and G. Marinescu for useful discussions.

\section{LOCALIZATION AND RESCALING OF THE PROBLEM}

In this section, we recall the results of [14, Sections 1.1 and 1.2] on the localization and rescaling of the problem, which allow us to obtain a formal asymptotic expansion of the renormalized Bochner Laplacian as $p \rightarrow \infty$.

We will keep the notation introduced in \$1. We fix $x_{0} \in X$. Let $\left\{e_{j}\right\}$ be an oriented orthonormal basis of $T_{x_{0}} X$. It gives rise to an isomorphism $X_{0}:=\mathbb{R}^{2 n} \cong T_{x_{0}} X$. Consider the trivial bundles $L_{0}$ and $E_{0}$ with fibers $L_{x_{0}}$ and $E_{x_{0}}$ on $X_{0}$. Recall that we have the Riemannian metric $g$ on $B^{T_{x_{0}} X}\left(0, a^{X}\right)$ as well as the connections $\nabla^{L}, \nabla^{E}$ and the Hermitian metrics $h^{L}, h^{E}$ on the restrictions of $L_{0}$ and $E_{0}$ to $B^{T_{x_{0}} X}\left(0, a^{X}\right)$ induced by the identification $B^{T_{x_{0}} X}\left(0, a^{X}\right) \cong B^{X}\left(x_{0}, a^{X}\right)$. In particular, $h^{L}, h^{E}$ are the constant metrics $h^{L_{0}}=h^{L_{x_{0}}}, h^{E_{0}}=h^{E_{x_{0}}}$. For $\varepsilon \in\left(0, a^{X} / 4\right)$, one can extend these geometric objects from $B^{T_{x_{0}} X}(0, \varepsilon)$ to $\mathbb{R}^{2 n} \cong T_{x_{0}} X$ in the following way. 
Let $\rho: \mathbb{R} \rightarrow[0,1]$ be a smooth even function such that $\rho(v)=1$ if $|v|<2$ and $\rho(v)=0$ if $|v|>4$. Let $\varphi_{\varepsilon}: \mathbb{R}^{2 n} \rightarrow \mathbb{R}^{2 n}$ be the map defined by $\varphi_{\varepsilon}(Z)=\rho(|Z| / \varepsilon) Z$. We equip $X_{0}$ with the metric $g_{0}(Z)=g\left(\varphi_{\varepsilon}(Z)\right)$. Set $\nabla^{E_{0}}=\varphi_{\varepsilon}^{*} \nabla^{E}$. Define the Hermitian connection $\nabla^{L_{0}}$ on $\left(L_{0}, h^{L_{0}}\right)$ by

$$
\nabla^{L_{0}}(Z)=\varphi_{\varepsilon}^{*} \nabla^{L}+\frac{1}{2}\left(1-\rho^{2}(|Z| / \varepsilon)\right) R_{x_{0}}^{L}(\mathcal{R}, \cdot)
$$

where $\mathcal{R}(Z)=\sum_{j} Z_{j} e_{j} \in \mathbb{R}^{2 n} \cong T_{Z} X_{0}$.

One can show that, for $\varepsilon$ small enough, the curvature $R^{L_{0}}$ is positive and satisfies the following estimate for any $x_{0} \in X$,

$$
\inf _{u \in T X} \frac{i R^{L_{0}}\left(u, J^{L_{0}} u\right)}{|u|_{g}^{2}} \geq(1-\alpha) \mu_{0} .
$$

From now on, we fix such an $\varepsilon>0$.

We also extend the function $\kappa$ from $B^{T_{x_{0}} X}(0, \varepsilon)$ to $X_{0}$. Let $d v_{X_{0}}$ be the Riemannian volume form of $\left(X_{0}, g_{0}\right)$. Then $\kappa$ is the smooth positive function on $X_{0}$ defined by the equation

$$
d v_{X_{0}}(Z)=\kappa(Z) d v_{T X}(Z), \quad Z \in X_{0} .
$$

Let $\Delta_{p}^{X_{0}}=\Delta^{L_{0}^{p} \otimes E_{0}}-p \tau_{0}$ be the associated renormalized Bochner Laplacian acting on $C^{\infty}\left(X_{0}, L_{0}^{p} \otimes E_{0}\right)$. Then (cf. [14, Equation (1.23)]) there exists a constant $C_{L_{0}}>0$ such that for any $p$ we have

$$
\sigma\left(\Delta_{p}^{X_{0}}\right) \subset\left[-C_{L_{0}}, C_{L_{0}}\right] \cup\left[2(1-\alpha) p \mu_{0}-C_{L_{0}},+\infty\right) .
$$

Consider the subspace $\mathcal{H}_{p}^{0}$ in $C^{\infty}\left(X_{0}, L_{0}^{p} \otimes E_{0}\right) \cong C^{\infty}\left(\mathbb{R}^{2 n}, E_{x_{0}}\right)$ spanned by the eigensections of $\Delta_{p}^{X_{0}}$ corresponding to eigenvalues in $\left[-C_{L_{0}}, C_{L_{0}}\right]$. Let $P_{\mathcal{H}_{p}^{0}}$ be the orthogonal projection onto $\mathcal{H}_{p}^{0}$. The smooth kernel of $\left(\Delta_{p}^{X_{0}}\right)^{q} P_{\mathcal{H}_{p}^{0}}$ with respect to the Riemannian volume form $d v_{X_{0}}$ is denoted by $P_{q, p}^{0}\left(Z, Z^{\prime}\right)$. The kernels $P_{q, p, x_{0}}\left(Z, Z^{\prime}\right)$ and $P_{q, p}^{0}\left(Z, Z^{\prime}\right)$ are asymptotically close on $B^{T_{x_{0}} X}(0, \varepsilon)$ in the $\mathcal{C}^{\infty}$-topology, as $p \rightarrow \infty$.

Proposition 1 ([14], Proposition 1.3). For any $l, m \in \mathbb{N}$, there exists $C_{l, m}>$ 0 such that for $x_{0} \in X$ and $x, x^{\prime} \in B^{T_{x_{0}} X}(0, \varepsilon)$, we have

$$
\left|P_{q, p, x_{0}}\left(Z, Z^{\prime}\right)-P_{q, p}^{0}\left(Z, Z^{\prime}\right)\right|_{\mathcal{C}^{m}} \leq C_{l, m} p^{-l} .
$$

Next, we use the rescaling introduced in [14, Section 1.2]. Denote $t=\frac{1}{\sqrt{p}}$. For $s \in C^{\infty}\left(\mathbb{R}^{2 n}, E_{x_{0}}\right)$, set

$$
S_{t} s(Z)=s(Z / t), \quad Z \in \mathbb{R}^{2 n} .
$$

The rescaled connection $\nabla_{t}$ is defined as

$$
\nabla_{t}=t S_{t}^{-1} \kappa^{\frac{1}{2}} \nabla^{L_{0}^{p}} \kappa^{-\frac{1}{2}} S_{t}
$$

Let $\Gamma^{L}, \Gamma^{E}$ be the connection forms of $\nabla^{L}, \nabla^{E}$ with respect to some fixed frames for $L$ and $E$ which are parallel along the curves $\gamma_{Z}:[0,1] \ni$ 
$u \rightarrow \exp _{x_{0}}^{X}(u Z)$ under the chosen trivializations on $B^{T_{x_{0}} X}(0, \varepsilon)$. Then on $B^{T_{x_{0}} X}(0, \varepsilon / t)$, we have

$$
\begin{aligned}
\nabla_{t, e_{i}} & =\kappa^{\frac{1}{2}}(t Z)\left(\nabla_{e_{i}}+\frac{1}{t} \Gamma^{L}\left(e_{i}\right)(t Z)+t \Gamma^{E}\left(e_{i}\right)(t Z)\right) \kappa^{-\frac{1}{2}}(t Z) \\
& =\nabla_{e_{i}}+\frac{1}{t} \Gamma^{L}\left(e_{i}\right)(t Z)+t \Gamma^{E}\left(e_{i}\right)(t Z)-t\left(\kappa^{-1}\left(e_{i} \kappa\right)\right)(t Z) .
\end{aligned}
$$

Recall that

$$
\sum_{|\alpha|=r}\left(\partial^{\alpha} \Gamma^{L}\right)_{x_{0}}\left(e_{j}\right) \frac{Z^{\alpha}}{\alpha !}=\frac{1}{r+1} \sum_{|\alpha|=r-1}\left(\partial^{\alpha} R^{L}\right)_{x_{0}}\left(\mathcal{R}, e_{j}\right) \frac{Z^{\alpha}}{\alpha !} .
$$

In particular,

$$
\Gamma^{L}\left(e_{j}\right)(Z)=\frac{1}{2} R_{x_{0}}^{L}\left(\mathcal{R}, e_{j}\right)+O\left(|Z|^{2}\right) .
$$

Similar identities are valid for $\Gamma^{E}$.

The rescaled operator $\mathcal{L}_{t}$ is defined to be

$$
\mathcal{L}_{t}=t^{2} S_{t}^{-1} \kappa^{\frac{1}{2}} \Delta_{p}^{X_{0}} \kappa^{-\frac{1}{2}} S_{t}
$$

We have

$$
\mathcal{L}_{t}=-g^{j k}(t Z)\left[\nabla_{t, e_{j}} \nabla_{t, e_{k}}-t \Gamma_{j k}^{\ell}(t Z) \nabla_{t, e_{\ell}}\right]-\tau(t Z) .
$$

By (44), it follows that

$$
\sigma\left(\mathcal{L}_{t}\right) \subset\left[-C_{L_{0}} t^{2}, C_{L_{0}} t^{2}\right] \cup\left[2(1-2 \alpha) \mu_{0}-C_{L_{0}},+\infty\right) .
$$

for sufficiently small $t$. Observe that the operators $\nabla_{t, e_{i}}$ and $\mathcal{L}_{t}$ depend smoothly on $t$ up to $t=0$. Their limits as $t \rightarrow 0$ are the operators

$$
\nabla_{0, e_{i}}=\nabla_{e_{i}}+\frac{1}{2} R_{x_{0}}^{L}\left(\mathcal{R}, e_{i}\right)
$$

and $\mathcal{L}_{0}$ given by (2). The spectrum of $\mathcal{L}_{0}$ consists of a discrete set of eigenvalues of infinite multiplicity (see, for instance, [14, Theorem 1.15]). In particular,

$$
\sigma\left(\mathcal{L}_{0}\right) \subset\{0\} \cup\left[2(1-2 \alpha) \mu_{0}-C_{L_{0}},+\infty\right) .
$$

One can develop the rescaled operator $\mathcal{L}_{t}$ in a Taylor series in $t$. For the resulting asymptotic expansion, we refer the reader to [14, Theorem 1.4].

\section{NORM ESTIMATES OF THE RESOLVENT}

The next step is the norm estimates. In this section, we derive the weighted norm estimates for the resolvent of the operator $\mathcal{L}_{t}$. First, we recall and slightly modify the results of [14, Section 1.3].

Denote by $C_{b}^{\infty}\left(\mathbb{R}^{2 n}, E_{x_{0}}\right)$ the space of smooth functions on $\mathbb{R}^{2 n}$ with values in $E_{x_{0}}$ whose derivatives of any order are uniformly bounded in $\mathbb{R}^{2 n}$. So $a \in C_{b}^{\infty}\left(\mathbb{R}^{2 n}, E_{x_{0}}\right)$ if, for any $\alpha \in \mathbb{Z}_{+}^{2 n}$, we have

$$
\sup _{Z \in \mathbb{R}^{2 n}}\left|\nabla_{e_{1}}^{\alpha_{1}} \ldots \nabla_{e_{2 n}}^{\alpha_{2 n}} a(Z)\right|<\infty
$$


For $m \in \mathbb{N}$ and $t>0$, let $\mathcal{Q}_{t}^{m}$ be the set of linear combinations of operators of the form $\nabla_{t, e_{i_{1}}} \ldots \nabla_{t, e_{i_{j}}}, j \leq m$, with coefficients from $C_{b}^{\infty}\left(\mathbb{R}^{2 n}, E_{x_{0}}\right)$. It is easy to see that $\mathcal{Q}_{t}^{m}$ is independent of $t$, therefore, we will omit $t$ in the notation: $\mathcal{Q}_{t}^{m}=\mathcal{Q}^{m}$. Observe that, if $Q$ is in $\mathcal{Q}^{m}$, then the adjoint $Q^{*}$ is in $\mathcal{Q}^{m}$.

For $s \in C_{c}^{\infty}\left(\mathbb{R}^{2 n}, E_{x_{0}}\right)$ set

$$
\|s\|_{t, 0}^{2}=\|s\|_{0}^{2}=\int_{\mathbb{R}^{2 n}}|s(Z)|^{2} d v_{T X}(Z) .
$$

and, for any $m \in \mathbb{N}$ and $t>0$,

$$
\|s\|_{t, m}^{2}=\sum_{\ell=0}^{m} \sum_{j_{1}, \ldots, j_{\ell}=1}^{2 n}\left\|\nabla_{t, e_{j_{1}}} \cdots \nabla_{t, e_{j_{\ell}}} s\right\|_{t, 0}^{2}
$$

Let $\langle\cdot, \cdot\rangle_{t, m}$ denote the inner product on $C^{\infty}\left(\mathbb{R}^{2 n}, E_{x_{0}}\right)$ corresponding to $\|\cdot\|_{t, m}^{2}$. Let $H_{t}^{m}$ be the Sobolev space of order $m$ with norm $\|\cdot\|_{t, m}$. For any integer $m<0$, we define the Sobolev space $H_{t}^{m}$ by duality. It is easy to see that, for different $t_{1}$ and $t_{2}$, the norms $\|\cdot\|_{t_{1}, m}$ and $\|\cdot\|_{t_{2}, m}$ are equivalent, uniformly in $t_{1}, t_{2} \in[0,1]$. For any bounded linear operator $A: H_{t}^{m} \rightarrow H_{t}^{m^{\prime}}$ with $m, m^{\prime} \in \mathbb{Z}$, we denote by $\|A\|_{t}^{m, m^{\prime}}$ its norm with respect to $\|\cdot\|_{t, m}$ and $\|\cdot\|_{t, m^{\prime}}$

Let $\delta$ be the circle in $\mathbb{C}$ oriented counterclockwise, centered at 0 and of radius $c \mu_{0}$. The following theorem is a slight modification of [14, Theorem $1.7]$.

Theorem 2. There exists $t_{0}>0$ such that the resolvent $\left(\lambda-\mathcal{L}_{t}\right)^{-1}$ exists for all $\lambda \in \delta, t \in\left[0, t_{0}\right]$. Moreover, there exists $C>0$ such that for all $\lambda \in \delta$, $t \in\left[0, t_{0}\right]$ and $x_{0} \in X$ we have

$\left\|\left(\lambda-\mathcal{L}_{t}\right)^{-1}\right\|_{t}^{0,0} \leq C / \mu_{0}, \quad\left\|\left(\lambda-\mathcal{L}_{t}\right)^{-1}\right\|_{t}^{0,1} \leq C / \sqrt{\mu_{0}}, \quad\left\|\left(\lambda-\mathcal{L}_{t}\right)^{-1}\right\|_{t}^{-1,1} \leq C$.

Proof. The first inequality follows by the spectral theorem. Next, we have

$$
\begin{aligned}
\left\|\left(\lambda-\mathcal{L}_{t}\right)^{-1} s\right\|_{t, 1}^{2} \leq \frac{1}{C_{1}}\left(\left\langle\mathcal{L}_{t}\left(\lambda-\mathcal{L}_{t}\right)^{-1} s,\left(\lambda-\mathcal{L}_{t}\right)^{-1} s\right\rangle_{t, 0}\right. & \\
& \left.+C_{2}\left\|\left(\lambda-\mathcal{L}_{t}\right)^{-1} s\right\|_{t, 0}^{2}\right) \leq \frac{C_{2}}{\mu_{0}}\|s\|_{t, 0}^{2} .
\end{aligned}
$$

For the third inequality, we refer to the proof of [14, Theorem 1.7].

Now we introduce weighted spaces. Consider a function $f \in C^{\infty}\left(\mathbb{R}^{2 n}\right)$ given by

$$
f(Z)=\left(1+|Z|^{2}\right)^{1 / 2}, \quad Z \in \mathbb{R}^{2 n} .
$$

An important point is that $f$ satisfies the estimates

$$
C_{1}|Z| \leq f(Z) \leq C_{2}|Z|, \quad|Z| \geq 1,
$$

with some $C_{1}, C_{2}>0$, and, for any $\alpha \in \mathbb{Z}_{+}^{2 n}$ with $|\alpha|>0$,

$$
\sup _{Z \in \mathbb{R}^{2 n}}\left|\nabla_{e_{1}}^{\alpha_{1}} \ldots \nabla_{e_{2 n}}^{\alpha_{2 n}} f(Z)\right|<\infty .
$$


For any $a \in \mathbb{R}$, let $L_{a}^{2}$ be the weighted $L^{2}$-space in $\mathbb{R}^{2 n}$ with the weight $e^{a f}$ :

$$
L_{a}^{2}=\left\{s: e^{a f} s \in L^{2}\left(\mathbb{R}^{2 n}, E_{x_{0}}\right)\right\} .
$$

A family $\left\{\|\cdot\|_{L_{a, W}^{2}}: W \in \mathbb{R}^{2 n}\right\}$ of equivalent norms in $L_{a}^{2}$ is defined by

$$
\|s\|_{L_{a, W}^{2}}^{2}=\left\|e^{a f_{W}} s\right\|_{0}^{2}=\int_{\mathbb{R}^{2 n}} e^{2 a f_{W}(Z)}|s(Z)|^{2} d v_{T X}(Z),
$$

where, for any $W \in \mathbb{R}^{2 n}$, the function $f_{W} \in C^{\infty}\left(\mathbb{R}^{2 n}\right)$ is given by

$$
f_{W}(Z)=f(Z-W)=\left(1+|Z-W|^{2}\right)^{1 / 2}, \quad Z \in \mathbb{R}^{2 n} .
$$

We will denote by $L_{a, W}^{2}$ the space $L_{a}^{2}$ with the norm $\|\cdot\|_{L_{a, W}^{2}}$. Similarly, one can introduce weighted Sobolev spaces.

Since the multiplication operator by $e^{a f_{W}}$ defines a unitary operator $e^{a f_{W}}$ : $L_{a, W}^{2} \rightarrow L^{2}$, any bounded operator $A$ in $L_{a, W}^{2}$ is unitarily equivalent to the operator $A_{a, W}=e^{a f_{W}} A e^{-a f_{W}}$ in $L^{2}$. In the sequel, instead of working directly with the weighted spaces $L_{a, W}^{2}$, we will consider operator families of the form $\left\{e^{a f_{W}} A e^{-a f_{W}}: W \in \mathbb{R}^{2 n}\right\}$ and only at the very end switch to weighted estimates.

First, we observe that

$$
\nabla_{t, a, W ; e_{j}}:=e^{a f_{W}} \nabla_{t, e_{j}} e^{-a f_{W}}=\nabla_{t, e_{j}}-a \nabla_{e_{j}} f_{W} .
$$

In particular, this immediately implies that, if $Q$ in $\mathcal{Q}^{m}$, then the operator $e^{a f_{W}} Q e^{-a f_{W}}$ is in $\mathcal{Q}^{m}$. Moreover, the family $\left\{e^{a f_{W}} Q e^{-a f_{W}}: W \in \mathbb{R}^{2 n}\right\}$ is a bounded family of operators belonging to $\mathcal{Q}^{m}$.

Next, for the operator $\mathcal{L}_{t, a, W}:=e^{a f_{W}} \mathcal{L}_{t} e^{-a f_{W}}$, we obtain

$$
\begin{aligned}
\mathcal{L}_{t, a, W} & =-g^{j k}(t Z)\left[\nabla_{t, a, W ; e_{j}} \nabla_{t, a, W ; e_{k}}-t \Gamma_{j k}^{\ell}(t Z) \nabla_{t, a, e_{\ell} ; W}\right]-\tau(t Z) \\
& =\mathcal{L}_{t}+a A_{W}+a^{2} B_{W}
\end{aligned}
$$

where

$$
\begin{aligned}
& A_{W}=-\sum_{j, k=1}^{2 n} g^{j k}(t Z)\left(\nabla_{e_{j}} f_{W} \nabla_{t, e_{k}}+\nabla_{e_{k}} f_{W} \nabla_{t, e_{j}}+\nabla_{e_{j}} \nabla_{e_{k}} f_{W}\right), \\
& B_{W}=-\sum_{j, k=1}^{2 n} g^{j k}(t Z) \nabla_{e_{j}} f_{W} \nabla_{e_{k}} f_{W} .
\end{aligned}
$$

In particular,

$$
\operatorname{Re} \mathcal{L}_{t, a, W}=\mathcal{L}_{t}-a^{2} \sum_{j, k=1}^{2 n} g^{j k}(t Z) \nabla_{e_{j}} f_{W} \nabla_{e_{k}} f_{W}=\mathcal{L}_{t}-a^{2}\left\|d f_{W}\right\|_{g^{-1}(t Z)}^{2}
$$

We have the following extension of [14, Theorem 1.6].

Theorem 3. There exist constants $C_{1}, C_{2}, C_{3}>0$ such that for any $t \in$ $[0,1], a \in \mathbb{R}, W \in \mathbb{R}^{2 n}$ and $s, s^{\prime} \in C_{c}^{\infty}\left(\mathbb{R}^{2 n}\right)$ we have

$$
\operatorname{Re}\left\langle\mathcal{L}_{t, a, W} s, s\right\rangle_{t, 0} \geq C_{1}\|s\|_{t, 1}^{2}-\left(C_{2}+C_{2}^{\prime} a^{2}\right)\|s\|_{t, 0}^{2},
$$




$$
\left|\left\langle\mathcal{L}_{t, a, W} s, s^{\prime}\right\rangle_{t, 0}\right| \leq C_{3}\left(\|s\|_{t, 1}\left\|s^{\prime}\right\|_{t, 1}+a^{2}\|s\|_{t, 0}\left\|s^{\prime}\right\|_{t, 0}\right) .
$$

Proof. Using (6), (8)), (9), and [14, Theorem 1.6], we get

$\operatorname{Re}\left\langle\mathcal{L}_{t, a, W} s, s\right\rangle_{t, 0} \geq\left\langle\mathcal{L}_{t} s, s\right\rangle_{t, 0}-C_{2}^{\prime} a^{2}\|s\|_{t, 0}^{2} \geq C_{1}\|s\|_{t, 1}^{2}-\left(C_{2}+C_{2}^{\prime} a^{2}\right)\|s\|_{t, 0}^{2}$ and

$$
\begin{aligned}
\left|\left\langle\mathcal{L}_{t, a, W} s, s^{\prime}\right\rangle_{t, 0}\right| & \leq\left|\left\langle\mathcal{L}_{t} s, s^{\prime}\right\rangle_{t, 0}\right|+|a|\left|\left\langle A_{W} s, s^{\prime}\right\rangle_{t, 0}\right|+a^{2}\left|\left\langle B_{W} s, s^{\prime}\right\rangle_{t, 0}\right| \\
& \leq C_{3}\left(\|s\|_{t, 1}\left\|s^{\prime}\right\|_{t, 1}+a^{2}\|s\|_{t, 0}\left\|s^{\prime}\right\|_{t, 0}\right) .
\end{aligned}
$$

Now we extend Theorem 2 to the operators $\mathcal{L}_{t, a, W}$.

Theorem 4. There exist $c>0$ and $C>0$ such that, for all $\lambda \in \delta, t \in\left[0, t_{0}\right]$, $|a|<c \sqrt{\mu_{0}}, W \in \mathbb{R}^{2 n}$ and $x_{0} \in X$ the inverse operator $\left(\lambda-\mathcal{L}_{t, a, W}\right)^{-1}$ exists and

$$
\left\|\left(\lambda-\mathcal{L}_{t, a, W}\right)^{-1}\right\|_{t}^{0,0} \leq C / \mu_{0}, \quad\left\|\left(\lambda-\mathcal{L}_{t, a, W}\right)^{-1}\right\|_{t}^{-1,1} \leq C .
$$

Proof. By Theorem [2, it follows that, for all $\lambda \in \delta, t \in\left[0, t_{0}\right], a \in \mathbb{R}$, $W \in \mathbb{R}^{2 n}$ and $x_{0} \in X$, we have

$$
\begin{aligned}
\left\|\left(\mathcal{L}_{t, a, W}-\mathcal{L}_{t}\right)\left(\lambda-\mathcal{L}_{t}\right)^{-1}\right\|_{t}^{0,0}=\left\|\left(a A_{W}+a^{2} B_{W}\right)\left(\lambda-\mathcal{L}_{t}\right)^{-1}\right\|_{t}^{0,0} \\
\leq C\left(a\left\|\left(\lambda-\mathcal{L}_{t}\right)^{-1}\right\|_{t}^{0,1}+a^{2}\left\|\left(\lambda-\mathcal{L}_{t}\right)^{-1}\right\|_{t}^{0,0}\right) \leq C\left(\frac{a}{\sqrt{\mu_{0}}}+\frac{a^{2}}{\mu_{0}}\right) .
\end{aligned}
$$

Similarly,

$$
\begin{aligned}
& \left\|\left(\mathcal{L}_{t, a, W}-\mathcal{L}_{t}\right)\left(\lambda-\mathcal{L}_{t}\right)^{-1}\right\|_{t}^{-1,0} \\
& \quad \leq C\left(a\left\|\left(\lambda-\mathcal{L}_{t}\right)^{-1}\right\|_{t}^{-1,1}+a^{2}\left\|\left(\lambda-\mathcal{L}_{t}\right)^{-1}\right\|_{t}^{-1,0}\right) \leq C\left(a+\frac{a^{2}}{\sqrt{\mu_{0}}}\right) .
\end{aligned}
$$

Choose $c>0$ such that $C\left(c+c^{2}\right)<\frac{1}{2}$. If $|a|<c \sqrt{\mu_{0}}$, then the operator $\lambda-\mathcal{L}_{t, a, W}$ is invertible in $L^{2}$. Using the resolvent identity

$$
\left(\lambda-\mathcal{L}_{t, a, W}\right)^{-1}=\left(\lambda-\mathcal{L}_{t}\right)^{-1}+\left(\lambda-\mathcal{L}_{t, a, W}\right)^{-1}\left(\mathcal{L}_{t, a, W}-\mathcal{L}_{t}\right)\left(\lambda-\mathcal{L}_{t}\right)^{-1},
$$

we infer

$$
\begin{aligned}
& \left\|\left(\lambda-\mathcal{L}_{t, a, W}\right)^{-1}\right\|_{t}^{0,0} \\
& \quad \leq\left\|\left(\lambda-\mathcal{L}_{t}\right)^{-1}\right\|_{t}^{0,0}+\left\|\left(\lambda-\mathcal{L}_{t, a, W}\right)^{-1}\right\|_{t}^{0,0}\left\|\left(\mathcal{L}_{t, a, W}-\mathcal{L}_{t}\right)\left(\lambda-\mathcal{L}_{t}\right)^{-1}\right\|_{t}^{0,0} \\
& \quad \leq\left\|\left(\lambda-\mathcal{L}_{t}\right)^{-1}\right\|_{t}^{0,0}+\frac{1}{2}\left\|\left(\lambda-\mathcal{L}_{t, a, W}\right)^{-1}\right\|_{t}^{0,0} .
\end{aligned}
$$

Therefore,

$$
\left\|\left(\lambda-\mathcal{L}_{t, a, W}\right)^{-1}\right\|_{t}^{0,0} \leq 2\left\|\left(\lambda-\mathcal{L}_{t}\right)^{-1}\right\|_{t}^{0,0} \leq C / \mu_{0} .
$$

Similarly,

$$
\left\|\left(\lambda-\mathcal{L}_{t, a, W}\right)^{-1}\right\|_{t}^{0,1}
$$




$$
\begin{aligned}
& \leq\left\|\left(\lambda-\mathcal{L}_{t}\right)^{-1}\right\|_{t}^{0,1}+\left\|\left(\lambda-\mathcal{L}_{t, a, W}\right)^{-1}\right\|_{t}^{0,1}\left\|\left(\mathcal{L}_{t, a}-\mathcal{L}_{t}\right)\left(\lambda-\mathcal{L}_{t}\right)^{-1}\right\|_{t}^{0,0} \\
& \leq\left\|\left(\lambda-\mathcal{L}_{t}\right)^{-1}\right\|_{t}^{0,1}+\frac{1}{2}\left\|\left(\lambda-\mathcal{L}_{t, a, W}\right)^{-1}\right\|_{t}^{0,1} .
\end{aligned}
$$

Therefore, we get

$$
\left\|\left(\lambda-\mathcal{L}_{t, a, W}\right)^{-1}\right\|_{t}^{0,1} \leq 2\left\|\left(\lambda-\mathcal{L}_{t}\right)^{-1}\right\|_{t}^{0,1} \leq C / \sqrt{\mu_{0}} .
$$

Finally,

$$
\begin{aligned}
& \left\|\left(\lambda-\mathcal{L}_{t, a, W}\right)^{-1}\right\|_{t}^{-1,1} \leq\left\|\left(\lambda-\mathcal{L}_{t}\right)^{-1}\right\|_{t}^{-1,1} \\
& \quad+\left\|\left(\lambda-\mathcal{L}_{t, a, W}\right)^{-1}\right\|_{t}^{0,1}\left\|\left(\mathcal{L}_{t, a, W}-\mathcal{L}_{t}\right)\left(\lambda-\mathcal{L}_{t}\right)^{-1}\right\|_{t}^{-1,0} \leq C .
\end{aligned}
$$

In the sequel, we will keep the notation $c$ for the constant given by Theorem 4. which will usually be related to the interval $\left(-c \sqrt{\mu_{0}}, c \sqrt{\mu_{0}}\right)$ of admissible values of the parameter $a$.

Observe that, for any $\lambda \in \delta, t \in\left[0, t_{0}\right],|a|<c \sqrt{\mu_{0}}, W \in \mathbb{R}^{2 n}$ and $x_{0} \in X$, the operators $\left(\lambda-\mathcal{L}_{t, a, W}\right)^{-1}$ and $\left(\lambda-\mathcal{L}_{t}\right)^{-1}$ are related by the identity

$$
\left(\lambda-\mathcal{L}_{t, a, W}\right)^{-1}=e^{a f_{W}}\left(\lambda-\mathcal{L}_{t}\right)^{-1} e^{-a f_{W}},
$$

which should be understood in the following way. If $a<0$, then, for any $s \in C_{c}^{\infty}\left(\mathbb{R}^{2 n}, E_{x_{0}}\right)$, the expression $e^{a f_{W}}\left(\lambda-\mathcal{L}_{t}\right)^{-1} e^{-a f_{W}} s$ makes sense and defines a function in $L^{2}\left(\mathbb{R}^{2 n}, E_{x_{0}}\right)$. Thus, we get a well defined operator

$$
e^{a f_{W}}\left(\lambda-\mathcal{L}_{t}\right)^{-1} e^{-a f_{W}}: C_{c}^{\infty}\left(\mathbb{R}^{2 n}, E_{x_{0}}\right) \rightarrow L^{2}\left(\mathbb{R}^{2 n}, E_{x_{0}}\right),
$$

one can check that $e^{a f_{W}}\left(\lambda-\mathcal{L}_{t}\right)^{-1} e^{-a f_{W}} s=\left(\lambda-\mathcal{L}_{t, a, W}\right)^{-1} s$ for any $s \in$ $C_{c}^{\infty}\left(\mathbb{R}^{2 n}, E_{x_{0}}\right)$. So (10) means that the operator $e^{a f_{W}}\left(\lambda-\mathcal{L}_{t}\right)^{-1} e^{-a f_{W}}$ extends to a bounded operator in $L^{2}\left(\mathbb{R}^{2 n}, E_{x_{0}}\right)$, which coincides with $(\lambda-$ $\left.\mathcal{L}_{t, a, W}\right)^{-1}$. If $a>0$, then, for any $s \in L^{2}\left(\mathbb{R}^{2 n}, E_{x_{0}}\right)$, the expression

$$
e^{a f_{W}}\left(\lambda-\mathcal{L}_{t}\right)^{-1} e^{-a f_{W}} s
$$

makes sense as a distribution on $\mathbb{R}^{2 n}$. Thus, we get a well defined operator

$$
e^{a f_{W}}\left(\lambda-\mathcal{L}_{t}\right)^{-1} e^{-a f_{W}}: L^{2}\left(\mathbb{R}^{2 n}, E_{x_{0}}\right) \rightarrow C^{-\infty}\left(\mathbb{R}^{2 n}, E_{x_{0}}\right) .
$$

So (10) means that this operator is indeed a bounded operator in $L^{2}\left(\mathbb{R}^{2 n}, E_{x_{0}}\right)$, which coincides with $\left(\lambda-\mathcal{L}_{t, a, W}\right)^{-1}$.

The following proposition is an extension of Proposition 1.8 in [14]

Proposition 2. For any natural $m$, there exists $C_{m}>0$ such that for any $t \in(0,1], Q_{1}, \ldots, Q_{m} \in\left\{\nabla_{t, a, W ; e_{i}}, Z_{i}\right\}_{i=1}^{2 n},|a|<c \sqrt{\mu_{0}}, W \in \mathbb{R}^{2 n}$ and $s, s^{\prime} \in C_{c}^{\infty}\left(\mathbb{R}^{2 n}, E_{x_{0}}\right)$ we have

$$
\left|\left\langle\left[Q_{1},\left[Q_{2}, \ldots,\left[Q_{m}, \mathcal{L}_{t, a, W}\right] \ldots\right]\right] s, s^{\prime}\right\rangle_{t, 0}\right| \leq C_{m}\|s\|_{t, 1}\left\|s^{\prime}\right\|_{t, 1}
$$

Proof. Recall the commutator relations

$$
\left[\nabla_{t, e_{i}}, Z_{j}\right]=\delta_{i j}, \quad\left[\nabla_{t, e_{i}}, \nabla_{t, e_{j}}\right]=R^{L_{0}}(t Z)\left(e_{i}, e_{j}\right) .
$$

It follows that

$$
\left[\nabla_{t, a, W ; e_{i}}, Z_{j}\right]=e^{a f_{W}}\left[\nabla_{t, e_{i}}, Z_{j}\right] e^{-a f_{W}}=\delta_{i j},
$$




$$
\left[\nabla_{t, a, W ; e_{i}}, \nabla_{t, a, W ; e_{j}}\right]=e^{a f_{W}}\left[\nabla_{t, e_{i}}, \nabla_{t, e_{j}}\right] e^{-a f_{W}}=R^{L_{0}}(t Z)\left(e_{i}, e_{j}\right) .
$$

By (77), the operator $\mathcal{L}_{t, a, W}$ has the form

$$
\mathcal{L}_{t, a, W}=\sum_{i, j} a_{i j}(t, t Z) \nabla_{t, a, W ; e_{i}} \nabla_{t, a, W ; e_{j}}+\sum_{i} b_{i}(t, t Z) \nabla_{t, a, W ; e_{i}}+c(t, t Z),
$$

where $a_{i j}(t, Z), b_{i}(t, Z), c(t, Z)$ as functions of $Z$ are in $C_{b}^{\infty}\left(\mathbb{R}^{2 n}, \operatorname{End}\left(E_{x_{0}}\right)\right)$ with all the norms uniformly bounded on $t \in[0,1]$. Moreover, they are polynomials in $t$. Using the commutator relations, one can see that, for $Q_{1}, \ldots, Q_{m} \in\left\{\nabla_{t, a, W ; e_{i}}, Z_{i}\right\}_{i=1}^{2 n}$, the operator $\left[Q_{1},\left[Q_{2}, \ldots,\left[Q_{m}, \mathcal{L}_{t, a, W}\right] \ldots\right]\right]$ has the same structure as $\mathcal{L}_{t, a, W}$. If $\left(\nabla_{t, a, W ; e_{i}}\right)^{*}$ is the adjoint of $\nabla_{t, a, W ; e_{i}}$ with respect to $\langle\cdot, \cdot\rangle_{t, 0}$, then

$$
\left(\nabla_{t, a, W ; e_{i}}\right)^{*}=-\nabla_{t, a, W ; e_{i}}-t\left(\kappa^{-1}\left(e_{i} \kappa\right)\right)(t Z)-2 a e_{i}\left(f_{W}\right)(Z) .
$$

Using these facts, one can easily complete the proof.

The following result is an analog of Theorem 1.9 in [14. For its proof, we can apply verbatim the proof of that theorem.

Theorem 5. For any $t \in\left(0, t_{0}\right], \lambda \in \delta, m \in \mathbb{N}, W \in \mathbb{R}^{2 n}$ and $|a|<c \sqrt{\mu_{0}}$, the resolvent $\left(\lambda-\mathcal{L}_{t, a, W}\right)^{-1}$ maps $H_{t}^{m}$ to $H_{t}^{m+1}$. Moreover, for any $\alpha \in \mathbb{Z}_{+}^{2 n}$ and $m \in \mathbb{N}$, there exists $C_{\alpha, m}>0$ such that for $t \in\left(0, t_{0}\right], \lambda \in \delta, W \in \mathbb{R}^{2 n}$ and $|a|<c \sqrt{\mu_{0}}$ we have

$$
\left\|Z^{\alpha}\left(\lambda-\mathcal{L}_{t, a, W}\right)^{-1} s\right\|_{t, m+1} \leq C_{\alpha, m} \sum_{\alpha^{\prime} \leq \alpha}\left\|Z^{\alpha^{\prime}} s\right\|_{t, m}, \quad s \in C_{c}^{\infty}\left(\mathbb{R}^{2 n}, E_{x_{0}}\right) .
$$

\section{Norm estimates of the Generalized Bergman projections}

In this section, we derive the weighted norm estimates for the generalized Bergman projection associated with the operator $\mathcal{L}_{t}$ and its derivatives of an arbitrary order with respect to $t$.

We let the symbol $\mathcal{P}_{0, t}$ stand for the spectral projection for $\mathcal{L}_{t}$ corresponding to the interval $\left[-C_{L_{0}} t^{2}, C_{L_{0}} t^{2}\right]$. Let $\mathcal{P}_{q, t}\left(Z, Z^{\prime}\right)=\mathcal{P}_{q, t, x_{0}}\left(Z, Z^{\prime}\right)$ be the smooth kernel of $\mathcal{P}_{q, t}=\left(\mathcal{L}_{t}\right)^{q} \mathcal{P}_{0, t}$ with respect to $d v_{T X}$. For any integers $k>0$ and $q \geq 0$, we can write

$$
\mathcal{P}_{q, t}=\left(\mathcal{L}_{t}\right)^{q} \mathcal{P}_{0, t}=\frac{1}{2 \pi i}\left(\begin{array}{c}
q+k-1 \\
k-1
\end{array}\right)^{-1} \int_{\delta} \lambda^{q+k-1}\left(\lambda-\mathcal{L}_{t}\right)^{-k} d \lambda .
$$

Proposition 3. For any $t \in\left(0, t_{0}\right], Q, Q^{\prime} \in \mathcal{Q}^{m}, W \in \mathbb{R}^{2 n}$ and $a,|a|<$ $c \sqrt{\mu_{0}}$, the operator $Q e^{a f_{W}} \mathcal{P}_{q, t} e^{-a f_{W}} Q^{\prime}$ extends to a bounded operator in $L^{2}\left(\mathbb{R}^{2 n}, E_{x_{0}}\right)$ with the norm bounded uniformly in $W$ and $t$.

Proof. First of all, we note that, for any $Q, Q^{\prime} \in \mathcal{Q}^{m}, W \in \mathbb{R}^{2 n}$, and $a \in \mathbb{R}$, the operator $Q e^{a f_{W}} \mathcal{P}_{q, t} e^{-a f_{W}} Q^{\prime}$ is well defined as an operator from $C_{c}^{\infty}\left(\mathbb{R}^{2 n}, E_{x_{0}}\right)$ to $C^{-\infty}\left(\mathbb{R}^{2 n}, E_{x_{0}}\right)$. 
By Theorem 5. it follows that, for any $Q \in \mathcal{Q}^{m}$, there exists $C_{m}>0$ such that, for all $t \in\left(0, t_{0}\right], \lambda \in \delta, W \in \mathbb{R}^{2 n}$, and $|a|<c \sqrt{\mu_{0}}$ we have

$$
\left\|Q\left(\lambda-\mathcal{L}_{t, a, W}\right)^{-m}\right\|_{t}^{0,0} \leq C_{m}
$$

Since $\mathcal{L}_{t}$ is formally self-adjoint with respect to $\|\cdot\|_{t, 0}$, we have $\mathcal{L}_{t, a, W}^{*}=$ $\mathcal{L}_{t,-a, W}$, so after taking the adjoints, for all $t \in\left(0, t_{0}\right], \lambda \in \delta, W \in \mathbb{R}^{2 n}$ and $|a|<c \sqrt{\mu_{0}}$ we get

$$
\left\|\left(\lambda-\mathcal{L}_{t, a, W}\right)^{-m} Q\right\|_{t}^{0,0} \leq C_{m} .
$$

Thus, for any $Q, Q^{\prime} \in \mathcal{Q}^{m}$, there exists $C_{m}>0$ such that, for all $t \in\left(0, t_{0}\right]$, $\lambda \in \delta, W \in \mathbb{R}^{2 n}$ and $|a|<c \sqrt{\mu_{0}}$ we have

$$
\left\|Q\left(\lambda-\mathcal{L}_{t, a, W}\right)^{-2 m} Q^{\prime}\right\|_{t}^{0,0} \leq C_{m}
$$

By the above estimates, the desired statement follows immediately from the formula

$$
\begin{aligned}
& Q e^{a f_{W}} \mathcal{P}_{q, t} e^{-a f_{W}} Q^{\prime} \\
& =\frac{1}{2 \pi i}\left(\begin{array}{c}
q+k-1 \\
k-1
\end{array}\right)^{-1} \int_{\delta} \lambda^{q+k-1} Q e^{a f_{W}}\left(\lambda-\mathcal{L}_{t}\right)^{-k} e^{-a f_{W}} Q^{\prime} d \lambda \\
& =\frac{1}{2 \pi i}\left(\begin{array}{c}
q+k-1 \\
k-1
\end{array}\right)^{-1} \int_{\delta} \lambda^{q+k-1} Q\left(\lambda-\mathcal{L}_{t, a, W}\right)^{-k} Q^{\prime} d \lambda
\end{aligned}
$$

with $k>2 m$, which can be justified much in the same way as relation (10) above.

Theorem 6. For any $r \geq 1, Q, Q^{\prime} \in \mathcal{Q}^{m}$, and $a,|a|<c \sqrt{\mu_{0}}$, there exists $C>0$ such that, for any $W \in \mathbb{R}^{2 n}$ and $t \in\left(0, t_{0}\right]$, we have

$$
\left\|Q e^{a f_{W}} \frac{\partial^{r}}{\partial t^{r}} \mathcal{P}_{q, t} e^{-a f_{W}} Q^{\prime} s\right\|_{t, 0} \leq C \sum_{|\beta| \leq 2 r}\left\|Z^{\beta} s\right\|_{t, 0}, \quad s \in C_{c}^{\infty}\left(\mathbb{R}^{2 n}, E_{x_{0}}\right) .
$$

Proof. We use the formula

$$
e^{a f_{W}} \frac{\partial^{r}}{\partial t^{r}} \mathcal{P}_{q, t} e^{-a f_{W}}=\frac{1}{2 \pi i}\left(\begin{array}{c}
q+k-1 \\
k-1
\end{array}\right)^{-1} \int_{\delta} \lambda^{q+k-1} \frac{\partial^{r}}{\partial t^{r}}\left(\lambda-\mathcal{L}_{t, a, W}\right)^{-k} d \lambda
$$

with $k>2(m+r+1)$.

Put

$$
I_{k, r}=\left\{(\mathbf{k}, \mathbf{r})=\left(k_{0}, \ldots, k_{j}, r_{1}, \ldots, r_{j}\right): \sum_{i=0}^{j} k_{i}=k, \sum_{i=1}^{j} r_{i}=r, k_{i}, r_{i} \in \mathbb{N}\right\} .
$$

Then we write

$$
\frac{\partial^{r}}{\partial t^{r}}\left(\lambda-\mathcal{L}_{t, a, W}\right)^{-k}=\sum_{(\mathbf{k}, \mathbf{r}) \in I_{k, r}} a_{\mathbf{r}}^{\mathbf{k}} A_{\mathbf{r}}^{\mathbf{k}}(\lambda, t, a, W),
$$

where the $a_{\mathbf{r}}^{\mathbf{k}}$ are some constants and

$$
A_{\mathbf{r}}^{\mathbf{k}}(\lambda, t, a, W)
$$




$$
=\left(\lambda-\mathcal{L}_{t, a, W}\right)^{-k_{0}} \frac{\partial^{r_{1}} \mathcal{L}_{t, a, W}}{\partial t^{r_{1}}}\left(\lambda-\mathcal{L}_{t, a, W}\right)^{-k_{1}} \cdots \frac{\partial^{r_{j}} \mathcal{L}_{t, a, W}}{\partial t^{r_{j}}}\left(\lambda-\mathcal{L}_{t, a, W}\right)^{-k_{j}} .
$$

Now we can proceed as in the proof of [14, Theorem 1.10]. We only observe that $\nabla_{t, a, W ; e_{j}}=\nabla_{t, e_{j}}-a \nabla_{e_{j}} f_{W}$, and, for $r>0$, we have $\frac{\partial^{r}}{\partial t^{r}} \nabla_{t, a, W ; e_{j}}=$ $\frac{\partial^{r}}{\partial t^{r}} \nabla_{t, e_{j}}$. We deduce that for any $Q, Q^{\prime} \in \mathcal{Q}^{m}, W \in \mathbb{R}^{2 n}$ and $a,|a|<c \sqrt{\mu_{0}}$, there exists $C>0$ such that

$$
\left\|Q A_{\mathbf{r}}^{\mathbf{k}}(\lambda, t, a, W) Q^{\prime} s\right\|_{t, 0} \leq C \sum_{|\beta| \leq 2 r}\left\|Z^{\beta} s\right\|_{t, 0}, \quad s \in C_{c}^{\infty}\left(\mathbb{R}^{2 n}, E_{x_{0}}\right),
$$

for $\lambda \in \delta$ and $t \in\left(0, t_{0}\right]$. This completes the proof.

Using similar arguments, one can prove the following theorem.

Theorem 7. For any $r \geq 1, Q, Q^{\prime} \in \mathcal{Q}^{m}, \alpha \in \mathbb{Z}_{+}^{2 n}$ and $a,|a|<c \sqrt{\mu_{0}}$, there exists $C>0$ such that, for any $W \in \mathbb{R}^{2 n}$ and $t \in\left(0, t_{0}\right]$, we have

$\left\|Q Z^{\alpha} e^{a f_{W}} \frac{\partial^{r}}{\partial t^{r}} \mathcal{P}_{q, t} e^{-a f_{W}} Q^{\prime} s\right\|_{t, 0} \leq C \sum_{|\beta| \leq|\alpha|+2 r}\left\|Z^{\beta} s\right\|_{t, 0}, \quad s \in C_{c}^{\infty}\left(\mathbb{R}^{2 n}, E_{x_{0}}\right)$.

\section{Asymptotic expansions And proofs of the main RESUlts}

In this section, we complete the proof of the main theorem. First, recall that, by [14, Theorem 1.11], for any $s \in C_{c}^{\infty}\left(\mathbb{R}^{2 n}, E_{x_{0}}\right)$, the limit of $\frac{\partial^{r}}{\partial t^{r}} \mathcal{P}_{q, t} s$ as $t \rightarrow 0$ in $L^{2}\left(\mathbb{R}^{2 n}, E_{x_{0}}\right)$ exists and

$$
\lim _{t \rightarrow 0} \frac{\partial^{r}}{\partial t^{r}} \mathcal{P}_{q, t} s=F_{q, r} s,
$$

where $F_{q, r}=F_{q, r, x_{0}}$ is the smoothing operator in $L^{2}\left(\mathbb{R}^{2 n}, E_{x_{0}}\right)$ given by

$$
F_{q, r}=\frac{1}{2 \pi i}\left(\begin{array}{c}
q+k-1 \\
k-1
\end{array}\right)^{-1} \int_{\delta} \lambda^{q+k-1} \sum_{(\mathbf{k}, \mathbf{r}) \in I_{k, r}} a_{\mathbf{r}}^{\mathbf{k}} A_{\mathbf{r}}^{\mathbf{k}}(\lambda, 0) d \lambda
$$

with $k$ sufficiently large, and

$$
A_{\mathbf{r}}^{\mathbf{k}}(\lambda, 0)=\left(\lambda-\mathcal{L}_{0}\right)^{-k_{0}} \frac{\partial^{r_{1}} \mathcal{L}_{t}}{\partial t^{r_{1}}}(0)\left(\lambda-\mathcal{L}_{0}\right)^{-k_{1}} \cdots \frac{\partial^{r_{j}} \mathcal{L}_{t}}{\partial t^{r_{j}}}(0)\left(\lambda-\mathcal{L}_{0}\right)^{-k_{j}} .
$$

Observe that the estimates in Theorem 7 are uniform in $t$ up to $t=$ 0 , which immediately implies that the same statement is fulfilled for the limiting value $t=0$. We conclude that, for any $r \geq 1, Q, Q^{\prime} \in \mathcal{Q}^{m}, \alpha \in \mathbb{Z}_{+}^{2 n}$ and $a,|a|<c \sqrt{\mu_{0}}$, there exists $C>0$ such that, for any $W \in \mathbb{R}^{2 n}$, we have

$$
\begin{aligned}
& \left\|Q Z^{\alpha} e^{a f_{W}} F_{q, r} e^{-a f_{W}} Q^{\prime} s\right\|_{0,0} \\
& \leq C \sum_{|\beta| \leq|\alpha|+2 r}\left\|Z^{\beta} s\right\|_{0,0}, \quad s \in C_{c}^{\infty}\left(\mathbb{R}^{2 n}, E_{x_{0}}\right) .
\end{aligned}
$$

For any $q$ and $j$, put

$$
R_{q, t}^{(j)}=P_{q, t}-\sum_{r=0}^{j} F_{q, r} t^{r}, \quad t>0 .
$$


Theorem 8. For any $Q, Q^{\prime} \in \mathcal{Q}^{m}, \alpha \in \mathbb{Z}_{+}^{2 n}$ and $a,|a|<c \sqrt{\mu_{0}}$, there exists $C>0$ such that, for any $W \in \mathbb{R}^{2 n}$ and $t \in\left[0, t_{0}\right]$, we have

$$
\left\|Q Z^{\alpha} e^{a f_{W}} R_{q, t}^{(j)} e^{-a f_{W}} Q^{\prime} s\right\|_{t, 0} \leq C t^{j+1} \sum_{|\beta| \leq|\alpha|+2 j+2}\left\|Z^{\beta} s\right\|_{t, 0}, \quad s \in C_{c}^{\infty}\left(\mathbb{R}^{2 n}, E_{x_{0}}\right) .
$$

Proof. The statement follows immediately from the Taylor formula

$$
\mathcal{P}_{q, t}-\sum_{r=0}^{j} \frac{1}{r !} F_{q, r} t^{r}=\frac{1}{j !} \int_{0}^{t}(t-\tau)^{j} \frac{\partial^{j+1} \mathcal{P}_{q, t}}{\partial t^{j+1}}(\tau) d \tau, \quad t \in[0,1],
$$

and estimates (12).

Theorem 9. For any $j, m, m^{\prime} \in \mathbb{N}, j \geq 2 q$, there exist $C>0$ and $M>0$ such that for any $0 \leq t \leq 1$ and $Z, Z^{\prime} \in T_{x_{0}} X$

$$
\begin{aligned}
\sup _{|\alpha|+\left|\alpha^{\prime}\right| \leq m} \mid & \left.\frac{\partial^{|\alpha|+\left|\alpha^{\prime}\right|}}{\partial Z^{\alpha} \partial Z^{\prime \alpha^{\prime}}}\left(\mathcal{P}_{q, t}\left(Z, Z^{\prime}\right)-\sum_{r=2 q}^{j} F_{q, r}\left(Z, Z^{\prime}\right) t^{r}\right)\right|_{\mathcal{C}^{m^{\prime}}(X)} \\
& \leq C t^{j+1}\left(1+|Z|+\left|Z^{\prime}\right|\right)^{M} \exp \left(-c \sqrt{\mu_{0}}\left|Z-Z^{\prime}\right|\right)+\mathcal{O}\left(t^{\infty}\right) .
\end{aligned}
$$

Proof. For $M \in \mathbb{N}$, let $\mathcal{D}^{M}$ be the set of differential operators in $\mathbb{R}^{2 n}$ of the form $\nabla_{e_{i_{1}}} \ldots \nabla_{e_{i_{j}}}$ with $j \leq M$. We claim that, for any $j \geq 2 q, D, D^{\prime} \in \mathcal{D}^{M}$ and for any $a,|a|<c \sqrt{\mu_{0}}$ there exists $C>0$ such that

$$
\left\|D e^{a f_{W}} R_{q, t}^{(j)} e^{-a f_{W}} D^{\prime} s\right\|_{t, 0} \leq C t^{j+1} \sum_{|\beta| \leq 2 j+2 M+2}\left\|Z^{\beta} s\right\|_{t, 0}
$$

for any $0 \leq t \leq 1$. To prove (14), we first observe that any $D \in \mathcal{D}^{M}$ can be written as

$$
D=\sum_{\alpha} A_{\alpha} Q_{\alpha}
$$

where $Q_{\alpha} \in \mathcal{Q}^{M}$ and the $A_{\alpha} \in C^{\infty}\left(\mathbb{R}^{2 n}, \operatorname{End}\left(E_{x_{0}}\right)\right)$ satisfy the following condition: for any $\beta \in \mathbb{Z}_{+}^{2 n}$, there exists $C_{\beta}>0$ such that

$$
\left|\nabla_{e_{1}}^{\beta_{1}} \ldots \nabla_{e_{2 n}}^{\beta_{2 n}} A_{\alpha}(Z)\right|<C_{\beta}(1+|Z|)^{M}, \quad Z \in \mathbb{R}^{2 n} .
$$

Similarly, any operator $D^{\prime} \in \mathcal{D}^{M}$ can be written as

$$
D^{\prime}=\sum_{\alpha^{\prime}} Q_{\alpha^{\prime}}^{\prime} A_{\alpha^{\prime}}^{\prime}
$$

where $Q_{\alpha^{\prime}}^{\prime} \in \mathcal{Q}^{M}$ and the $A_{\alpha^{\prime}}^{\prime} \in C^{\infty}\left(\mathbb{R}^{2 n}, \operatorname{End}\left(E_{x_{0}}\right)\right)$ satisfy (15).

Then we have

$$
\left\|D e^{a f_{W}} R_{q, t}^{(j)} e^{-a f_{W}} D^{\prime} s\right\|_{t, 0} \leq \sum_{\alpha, \alpha^{\prime}}\left\|A_{\alpha} Q_{\alpha} e^{a f_{W}} R_{q, t}^{(j)} e^{-a f_{W}} Q_{\alpha^{\prime}}^{\prime} A_{\alpha^{\prime}}^{\prime} s\right\|_{t, 0} .
$$

For every term in the right-hand side of the last inequality, we get

$$
\left\|A_{\alpha} Q_{\alpha} e^{a f_{W}} R_{q, t}^{(j)} e^{-a f_{W}} Q_{\alpha^{\prime}}^{\prime} A_{\alpha^{\prime}}^{\prime} s\right\|_{t, 0}
$$




$$
\begin{aligned}
& \leq C\left\|(1+|Z|)^{m} Q_{\alpha} e^{a f_{W}} R_{q, t}^{(j)} e^{-a f_{W}} Q_{\alpha^{\prime}}^{\prime} A_{\alpha^{\prime}}^{\prime} s\right\|_{t, 0} \\
& \leq C t^{j+1} \sum_{|\beta| \leq 2 j+M+2}\left\|Z^{\beta} A_{\alpha^{\prime}}^{\prime} s\right\|_{t, 0} \\
& \leq C t^{j+1} \sum_{|\beta| \leq 2 j+2 M+2}\left\|Z^{\beta} s\right\|_{t, 0},
\end{aligned}
$$

which completes the proof of (14).

Let $H^{m}\left(\mathbb{R}^{2 n}, E_{x_{0}}\right)$ denote the usual Sobolev space on $\mathbb{R}^{2 n}$ with the norm

$$
\|u\|_{H^{m}\left(\mathbb{R}^{2 n}, E_{x_{0}}\right)}=\left(\int_{\mathbb{R}^{2 n}}\left(1+|\xi|^{2}\right)^{m / 2}|\hat{u}(\xi)|^{2}\right)^{1 / 2}
$$

$\hat{u}$ is the Fourier transform of $u$. By (14), it follows that, for any $m, m^{\prime} \in \mathbb{R}$ and $a,|a|<c \sqrt{\mu_{0}}$ there exist $M \in \mathbb{N}$ and $C>0$ such that

$$
\left\|e^{a f_{W}} R_{q, t}^{(j)} e^{-a f_{W}} s\right\|_{H^{m}\left(\mathbb{R}^{2 n}, E_{x_{0}}\right)} \leq C t^{j+1} \sum_{|\beta| \leq M}\left\|Z^{\beta} s\right\|_{H^{m^{\prime}}\left(\mathbb{R}^{2 n}, E_{x_{0}}\right)}
$$

for any $0<t \leq 1$ and $s \in C_{c}^{\infty}\left(\mathbb{R}^{2 n}, E_{x_{0}}\right)$. In particular, for any $s \in$ $H^{m^{\prime}}\left(\mathbb{R}^{2 n}, E_{x_{0}}\right)$ with supp $s \subset B(0, \sigma)$ for some $\sigma>0$, we have

$$
\left\|e^{a f_{W}} R_{q, t}^{(j)} e^{-a f_{W}} s\right\|_{H^{m}\left(\mathbb{R}^{2 n}, E_{x_{0}}\right)} \leq C t^{j+1}(1+\sigma)^{M}\|s\|_{H^{m^{\prime}}\left(\mathbb{R}^{2 n}, E_{x_{0}}\right)} .
$$

For any $v \in E_{x_{0}}$, consider the delta-function $\delta_{0}^{v} \in C^{-\infty}\left(\mathbb{R}^{2 n}, E_{x_{0}}\right)$ defined by $\left\langle\delta_{0}^{v}, s\right\rangle=\langle v, s(0)\rangle_{h^{E x_{0}}}$ for $s \in C_{c}^{\infty}\left(\mathbb{R}^{2 n}, E_{x_{0}}\right)$. Let $\delta_{Z^{\prime}}^{v} \in C^{-\infty}\left(\mathbb{R}^{2 n}, E_{x_{0}}\right)$ be the delta-function at $Z^{\prime} \in \mathbb{R}^{2 n}: \delta_{Z^{\prime}}^{v}(Z)=\delta_{0}^{v}\left(Z-Z^{\prime}\right)$. Then $\delta_{Z^{\prime}}^{v} \in$ $H^{-(n+1)}\left(\mathbb{R}^{2 n}, E_{x_{0}}\right)$, with the norm, uniformly bounded in $Z^{\prime}$ and $v$ with $|v|_{h^{E x_{0}}}=1$ (actually, independent of $Z^{\prime}$ ). We can write

$$
\frac{\partial^{\left|\alpha^{\prime}\right|}}{\partial Z^{\prime \alpha^{\prime}}}\left(e^{a f_{W}(Z)} R_{q, t}^{(j)}\left(Z, Z^{\prime}\right) e^{-a f_{W}\left(Z^{\prime}\right)} v\right)=\left(e^{a f_{W}} R_{q, t}^{(j)} e^{-a f_{W}} D_{\alpha^{\prime}}^{\prime} \delta_{Z^{\prime}}^{v}\right)
$$

with some $D_{\alpha^{\prime}}^{\prime} \in \mathcal{D}^{m}$. Therefore, we get

$$
\begin{gathered}
\sup _{|\alpha|+\left|\alpha^{\prime}\right| \leq m}\left|\frac{\partial^{|\alpha|+\left|\alpha^{\prime}\right|}}{\partial Z^{\alpha} \partial Z^{\prime \alpha^{\prime}}}\left(e^{a f_{W}(Z)} R_{q, t}^{(j)}\left(Z, Z^{\prime}\right) e^{-a f_{W}\left(Z^{\prime}\right)} v\right)\right| \\
\leq C \sup _{\left|\alpha^{\prime}\right| \leq m}\left\|\frac{\partial^{\left|\alpha^{\prime}\right|}}{\partial Z^{\prime \alpha^{\prime}}}\left(e^{a f_{W}(Z)} R_{q, t}^{(j)}\left(Z, Z^{\prime}\right) e^{-a f_{W}\left(Z^{\prime}\right)} v\right)\right\|_{C_{b}^{m}\left(\mathbb{R}^{2 n}, E_{x_{0}}\right)} \\
=C \sum_{\alpha^{\prime}}\left\|e^{a f_{W}} R_{q, t}^{(j)} e^{-a f_{W}} D_{\alpha^{\prime}}^{\prime} \delta_{Z^{\prime}}^{v}\right\|_{C_{b}^{m}\left(\mathbb{R}^{2 n}, E_{x_{0}}\right)}
\end{gathered}
$$

By the Sobolev embedding theorem, $H^{M}\left(\mathbb{R}^{2 n}, E_{x_{0}}\right) \hookrightarrow C_{b}^{m}\left(\mathbb{R}^{2 n}, E_{x_{0}}\right)$ for $M=m+n+1$. Therefore, using (16), for $\left|Z^{\prime}\right| \leq \sigma$ with an arbitrary $\sigma>0$ we get

$$
\left\|e^{a f_{W}} R_{q, t}^{(j)} e^{-a f_{W}} D_{\alpha^{\prime}}^{\prime} \delta_{Z^{\prime}}^{v}\right\|_{C_{b}^{m}\left(\mathbb{R}^{2 n}, E_{x_{0}}\right)}
$$




$$
\begin{aligned}
& \leq C\left\|e^{a f_{W}} R_{q, t}^{(j)} e^{-a f_{W}} D_{\alpha^{\prime}}^{\prime} \delta_{Z^{\prime}}^{v}\right\|_{H^{m+n+1}\left(\mathbb{R}^{2 n}, E_{x_{0}}\right)} \\
& \leq C t^{j+1}(1+\sigma)^{2 j+2 m+2 n+4}\left\|\delta_{Z^{\prime}}^{v}\right\|_{H^{-(m+n+1)}\left(\mathbb{R}^{2 n}, E_{x_{0}}\right)} .
\end{aligned}
$$

Setting $W=Z^{\prime}$, we obtain

$$
\sup _{|\alpha|+\left|\alpha^{\prime}\right| \leq m}\left|\frac{\partial^{|\alpha|+\left|\alpha^{\prime}\right|}}{\partial Z^{\alpha} \partial Z^{\prime \alpha^{\prime}}}\left(e^{a f_{Z^{\prime}}(Z)} R_{q, t}^{(j)}\left(Z, Z^{\prime}\right)\right)\right| \leq C t^{j+1}(1+\sigma)^{2 j+2 m+2 n+4}
$$

and

$$
\begin{aligned}
\sup _{|\alpha|+\left|\alpha^{\prime}\right| \leq m}\left|\frac{\partial^{|\alpha|+\left|\alpha^{\prime}\right|}}{\partial Z^{\alpha} \partial Z^{\prime \alpha^{\prime}}} R_{q, t}^{(j)}\left(Z, Z^{\prime}\right)\right| & \leq C t^{j+1}(1+\sigma)^{2 j+2 m+2 n+4} e^{-a f_{Z^{\prime}}(Z)} \\
& \leq C t^{j+1}\left(1+\left|Z^{\prime}\right|\right)^{2 j+2 m+2 n+4} e^{-a\left|Z-Z^{\prime}\right|}
\end{aligned}
$$

which completes the proof in the case $m^{\prime}=0$.

To treat the case where $m^{\prime} \geq 1$, we proceed as in the proof of [14, Theorem 1.10]. For any vector $U \in T_{x_{0}} X$, the following formula holds:

$$
\nabla_{U} \mathcal{P}_{q, t}=\left(\mathcal{L}_{t}\right)^{q} \mathcal{P}_{0, t}=\frac{1}{2 \pi i}\left(\begin{array}{c}
q+k-1 \\
k-1
\end{array}\right)^{-1} \int_{\delta} \lambda^{q+k-1} \nabla_{U}\left(\lambda-\mathcal{L}_{t}\right)^{-k} d \lambda
$$

The operator $\nabla_{U}\left(\lambda-\mathcal{L}_{t}\right)^{-k}$ is given by a formula similar to (11). Then we observe that $\nabla_{U} \mathcal{L}_{t}$ is a differential operator on $T_{x_{0}} X$ with the same structure as $\mathcal{L}_{t}$. This allows us to extend all our considerations to the case of an arbitrary $m^{\prime} \geq 1$.

To complete the proof of Theorem 1] we observe that, by (5),

$$
P_{q, p}^{0}\left(Z, Z^{\prime}\right)=t^{-2 n-2 q} \kappa^{-\frac{1}{2}}(Z) \mathcal{P}_{q, t}\left(Z / t, Z^{\prime} / t\right) \kappa^{-\frac{1}{2}}\left(Z^{\prime}\right), \quad Z, Z^{\prime} \in \mathbb{R}^{2 n},
$$

and make use of Proposition 1. We remark that, by [14, Theorem 1.18], we have $F_{q, r}=0$ for $q>0, r<2 q$.

\section{TOeplitz operators}

In this section, we construct the algebra of Toeplitz operators associated with the renormalized Bochner Laplacian on the symplectic manifold $X$. The proofs of the results of this section are obtained by a word for word repetition of the arguments of the paper [15]. So we only give basic definitions and statements of the main results. As mentioned in the Introduction, the algebra of Toeplitz operators associated with the renormalized Bochner Laplacian was also constructed in [8].

Definition 1. A Toeplitz operator is a sequence $\left\{T_{p}\right\}=\left\{T_{p}\right\}_{p \in \mathbb{N}}$ of bounded linear operators $T_{p}: L^{2}\left(X, L^{p} \otimes E\right) \rightarrow L^{2}\left(X, L^{p} \otimes E\right)$, satisfying the following conditions.

(i): For any $p \in \mathbb{N}$, we have

$$
T_{p}=P_{\mathcal{H}_{p}} T_{p} P_{\mathcal{H}_{p}} .
$$


(ii): There exists a sequence $g_{l} \in C^{\infty}(X, \operatorname{End}(E))$ such that

$$
T_{p}=P_{\mathcal{H}_{p}}\left(\sum_{l=0}^{\infty} p^{-l} g_{l}\right) P_{\mathcal{H}_{p}}+\mathcal{O}\left(p^{-\infty}\right)
$$

i.e. for any natural $k$ there exists $C_{k}>0$ such that

$$
\left\|T_{p}-P_{\mathcal{H}_{p}}\left(\sum_{l=0}^{k} p^{-l} g_{l}\right) P_{\mathcal{H}_{p}}\right\| \leq C_{k} p^{-k-1} .
$$

The full symbol of $\left\{T_{p}\right\}$ is the formal series $\sum_{l=0}^{\infty} \hbar^{l} g_{l} \in C^{\infty}(X, \operatorname{End}(E))[[\hbar]]$ and the principal symbol of $\left\{T_{p}\right\}$ is $g_{0}$.

In the particular case when $g_{l}=0$ for $l \geq 1$ and $g_{0}=f$, we get the operator $T_{f, p}=P_{\mathcal{H}_{p}} f P_{\mathcal{H}_{p}}: L^{2}\left(X, L^{p} \otimes E\right) \rightarrow L^{2}\left(X, L^{p} \otimes E\right)$. The Schwartz kernel of $T_{f, p}$ is given by

$$
T_{f, p}\left(x, x^{\prime}\right)=\int_{X} P_{p}\left(x, x^{\prime \prime}\right) f\left(x^{\prime \prime}\right) P_{p}\left(x^{\prime \prime}, x^{\prime}\right) d v_{X}\left(x^{\prime \prime}\right) .
$$

Lemma 1. For any $\varepsilon>0$ and $l, m \in \mathbb{N}$, there exists $C>0$ such that for any $p \geq 1$ and $\left(x, x^{\prime}\right) \in X \times X$ with $d\left(x, x^{\prime}\right)>\varepsilon$ we have

$$
\left|T_{f, p}\left(x, x^{\prime}\right)\right|_{C^{m}} \leq C p^{-l} \text {. }
$$

Let $\left\{\Xi_{p}\right\}$ be a sequence of linear operators $\Xi_{p}: L^{2}\left(X, L^{p} \otimes E\right) \rightarrow L^{2}\left(X, L^{p} \otimes\right.$ $E)$ with smooth kernel $\Xi_{p}\left(x, x^{\prime}\right)$ with respect to $d v_{X}$. As described in the Introduction, $\Xi_{p}\left(x, x^{\prime}\right)$ induces a smooth section $\Xi_{p, x_{0}}\left(Z, Z^{\prime}\right)$ of the vector bundle $\pi^{*}(\operatorname{End}(E))$ on $T X \times_{X} T X$ defined for all $x_{0} \in X$ and $Z, Z^{\prime} \in T_{x_{0}} X$ with $|Z|,\left|Z^{\prime}\right|<a_{X}$. Recall that $\mathcal{P}=\mathcal{P}_{x_{0}}$ denotes the Bergman kernel in $\mathbb{R}^{2 n}$ given by (1).

Definition 2. We say that

$$
p^{-n} \Xi_{p, x_{0}}\left(Z, Z^{\prime}\right) \cong \sum_{r=0}^{k}\left(Q_{r, x_{0}} \mathcal{P}_{x_{0}}\right)\left(\sqrt{p} Z, \sqrt{p} Z^{\prime}\right) p^{-\frac{r}{2}}+\mathcal{O}\left(p^{-\frac{k+1}{2}}\right)
$$

with some $Q_{r, x_{0}} \in \operatorname{End}\left(E_{x_{0}}\right)\left[Z, Z^{\prime}\right], 0 \leq r \leq k$, depending smoothly on $x_{0} \in X$, if there exist $\varepsilon^{\prime} \in\left(0, a_{X}\right]$ and $C_{0}>0$ with the following property: for any $l \in \mathbb{N}$, there exist $C>0$ and $M>0$ such that for any $x_{0} \in X, p \geq 1$ and $Z, Z^{\prime} \in T_{x_{0}} X,|Z|,\left|Z^{\prime}\right|<\varepsilon^{\prime}$, we have

$$
\begin{aligned}
& \left|p^{-n} \Xi_{p, x_{0}}\left(Z, Z^{\prime}\right) \kappa^{\frac{1}{2}}(Z) \kappa^{\frac{1}{2}}\left(Z^{\prime}\right)-\sum_{r=0}^{k}\left(Q_{r, x_{0}} \mathcal{P}_{x_{0}}\right)\left(\sqrt{p} Z, \sqrt{p} Z^{\prime}\right) p^{-\frac{r}{2}}\right|_{\mathcal{C}^{l}(X)} \\
& \quad \leq C p^{-\frac{k+1}{2}}\left(1+\sqrt{p}|Z|+\sqrt{p}\left|Z^{\prime}\right|\right)^{M} \exp \left(-\sqrt{C_{0} p}\left|Z-Z^{\prime}\right|\right)+\mathcal{O}\left(p^{-\infty}\right) .
\end{aligned}
$$

By Theorem 1, for any $k \in \mathbb{N}$, we have

$$
p^{-n} P_{p, x_{0}}\left(Z, Z^{\prime}\right) \cong \sum_{r=0}^{k}\left(F_{0, r, x_{0}} \mathcal{P}_{x_{0}}\right)\left(\sqrt{p} Z, \sqrt{p} Z^{\prime}\right) p^{-\frac{r}{2}}+\mathcal{O}\left(p^{-\frac{k+1}{2}}\right) \text {. }
$$


For any polynomial $F \in \mathbb{C}\left[Z, Z^{\prime}\right]$, consider the operator $F \mathcal{P}$ in $L^{2}\left(T_{x_{0}} X\right) \cong$ $L^{2}\left(\mathbb{R}^{2 n}\right)$ with the kernel $(F \mathcal{P})\left(Z, Z^{\prime}\right)$ with respect to $d Z$. For any polynomials $F, G \in \mathbb{C}\left[Z, Z^{\prime}\right]$, define the polynomial $\mathcal{K}[F, G] \in \mathbb{C}\left[Z, Z^{\prime}\right]$ by the condition

$$
((F \mathcal{P}) \circ(G \mathcal{P}))\left(Z, Z^{\prime}\right)=(\mathcal{K}[F, G] \mathcal{P})\left(Z, Z^{\prime}\right),
$$

where $(F \mathcal{P}) \circ(G \mathcal{P})$ is the composition of the operators $F \mathcal{P}$ and $G \mathcal{P}$ in $L^{2}\left(T_{x_{0}} X\right)$.

Lemma 2. Let $f \in C^{\infty}(X, \operatorname{End}(E))$. For any $k \in \mathbb{N}, x_{0} \in X, Z, Z^{\prime} \in T_{x_{0}} X$, $|Z|,\left|Z^{\prime}\right|<\varepsilon / 2$, we have

$$
p^{-n} T_{f, p, x_{0}}\left(Z, Z^{\prime}\right) \cong \sum_{r=0}^{k}\left(Q_{r, x_{0}}(f) \mathcal{P}_{x_{0}}\right)\left(\sqrt{p} Z, \sqrt{p} Z^{\prime}\right) p^{-\frac{r}{2}}+\mathcal{O}\left(p^{-\frac{k+1}{2}}\right),
$$

where the polynomials $Q_{r, x_{0}}(f) \in \operatorname{End}\left(E_{x_{0}}\right)\left[Z, Z^{\prime}\right]$ have the same parity as $r$ and are given by

$$
Q_{r, x_{0}}(f)=\sum_{r_{1}+r_{2}+|\alpha|=r} \mathcal{K}\left[F_{0, r_{1}, x_{0}}, \frac{\partial^{\alpha} f_{x_{0}}}{\partial Z^{\alpha}}(0) \frac{Z^{\alpha}}{\alpha !} F_{0, r_{2}, x_{0}}\right],
$$

In particular,

$$
\begin{aligned}
& Q_{0, x_{0}}(f)=f\left(x_{0}\right), \\
& Q_{1, x_{0}}(f)=f\left(x_{0}\right) F_{0,1, x_{0}}+\mathcal{K}\left[F_{0,0, x_{0}}, \frac{\partial f_{x_{0}}}{\partial Z_{j}}(0) Z_{j} F_{0,0, x_{0}}\right] .
\end{aligned}
$$

We have the following criterion for Toeplitz operators.

Theorem 10. A family $\left\{T_{p}: L^{2}\left(X, L^{p} \otimes E\right) \rightarrow L^{2}\left(X, L^{p} \otimes E\right)\right\}$ of bounded linear operators is a Toeplitz operator if and only if it satisfies the following three conditions:

(i): For any $p \in \mathbb{N}$, we have

$$
T_{p}=P_{\mathcal{H}_{p}} T_{p} P_{\mathcal{H}_{p}} .
$$

(ii): For any $\varepsilon_{0}>0$ and $l \in \mathbb{N}$, there exists $C>0$ such that

$$
\left|T_{f, p}\left(x, x^{\prime}\right)\right| \leq C p^{-l}
$$

for any $p \geq 1$ and $\left(x, x^{\prime}\right) \in X \times X$ with $d\left(x, x^{\prime}\right)>\varepsilon_{0}$.

(iii): There exist a family of polynomials $\mathcal{Q}_{r, x_{0}} \in \operatorname{End}\left(E_{x_{0}}\right)\left[Z, Z^{\prime}\right]$, depending smoothly on $x_{0}$, of the same parity as $r$ and $\varepsilon^{\prime} \in\left(0, a_{X} / 4\right)$ such that

$p^{-n} T_{p, x_{0}}\left(Z, Z^{\prime}\right) \cong \sum_{r=0}^{k}\left(\mathcal{Q}_{r, x_{0}} \mathcal{P}_{x_{0}}\right)\left(\sqrt{p} Z, \sqrt{p} Z^{\prime}\right) p^{-\frac{r}{2}}+\mathcal{O}\left(p^{-\frac{k+1}{2}}\right)$

for any $k \in \mathbb{N}, x_{0} \in X, Z, Z^{\prime} \in T_{x_{0}} X,|Z|,\left|Z^{\prime}\right|<\varepsilon^{\prime}$.

Using this criterion, one can show that the set of Toeplitz operators is an algebra. 
Theorem 11. Let $f, g \in C^{\infty}(X, \operatorname{End}(E))$. Then the composition of the Toeplitz operators $T_{f, p}$ and $T_{g, p}$ is a Toeplitz operator. More precisely, it admits the asymptotic expansion

$$
T_{f, p} T_{g, p}=\sum_{r=0}^{\infty} p^{-r} T_{C_{r}(f, g), p}+\mathcal{O}\left(p^{-\infty}\right),
$$

with some $C_{r}(f, g) \in C^{\infty}(X$, End $(E))$, where the $C_{r}$ are bidifferential operators. In particular, $C_{0}(f, g)=f g$ and, for $f, g \in C^{\infty}(X)$, we have

$$
C_{1}(f, g)-C_{1}(f, g)=i\{f, g\}
$$

where $\{f, g\}$ is the Poisson bracket on $(X, 2 \pi \omega)$.

\section{REFERENCES}

[1] Meladze, G. A.; Shubin, M. A. Proper uniform pseudodifferential operators on unimodular Lie groups. (Russian). Tr. Sem. Petrovsk. No. 11 (1986), 74-97; English transl., J. Soviet Math. 45 (1989), no. 5, 1421-1439.

[2] Meladze, G. A.; Shubin, M. A. A functional calculus of pseudodifferential operators on unimodular Lie groups. (Russian). Tr. Sem. Petrovsk. No. 12 (1987), 164-200; English transl., J. Soviet Math. 47 (1989), no. 4, 2607-2638.

[3] Bismut, J.-M.; Vasserot, E. The asymptotics of the Ray-Singer analytic torsion associated with high powers of a positive line bundle. Comm. Math. Phys. 125 (1989), 355-367.

[4] Borthwick, D.; Uribe, A. Almost complex structures and geometric quantization. Math. Res. Lett. 3 (1996), 845-861.

[5] Braverman, M. Vanishing theorems on covering manifolds. Tel Aviv Topology Conference: Rothenberg Festschrift (1998), 1-23, Contemp. Math., 231, Amer. Math. Soc., Providence, RI, 1999.

[6] Dai, X.; Liu, K.; Ma, X. On the asymptotic expansion of Bergman kernel. J. Differential Geom. 72 (2006), 1-41.

[7] Guillemin, V.; Uribe, A. The Laplace operator on the $n$th tensor power of a line bundle: eigenvalues which are uniformly bounded in $n$. Asymptotic Anal. 1 (1988), $105-113$.

[8] Ioos, L.; Lu, W.; Ma, X.; Marinescu, G. Berezin-Toeplitz quantization for eigenstates of the Bochner-Laplacian on symplectic manifolds, preprint arXiv:1703.06420.

[9] Kordyukov, Yu. A. $L^{p}$-theory of elliptic differential operators on manifolds of bounded geometry. Acta Appl. Math. 23 (1991), no. 3, 223-260.

[10] Kordyukov, Yu. A. $L^{p}$-estimates for functions of elliptic operators on manifolds of bounded geometry. Russ. J. Math. Phys. 7 (2000), no. 2, 216-229.

[11] Lu, W.; Ma, X.; Marinescu, G. Donaldson's Q-operators for symplectic manifolds, Sci. China Math. 60 (2017), 1047-1056.

[12] Ma, X.; Marinescu, G. The $\operatorname{Spin}^{c}$ Dirac operator on high tensor powers of a line bundle. Math. Z. 240 (2002), 651-664.

[13] Ma, X.; Marinescu, G. Holomorphic Morse inequalities and Bergman kernels. Progress in Mathematics, 254. Birkhäuser Verlag, Basel, 2007.

[14] Ma, X.; Marinescu, G. Generalized Bergman kernels on symplectic manifolds. Adv. Math. 217 (2008), 1756-1815.

[15] Ma, X.; Marinescu, G. Toeplitz operators on symplectic manifolds. J. Geom. Anal. 18 (2008), 565-611. 
Institute of Mathematics with Computing Centre, Ufa Federal Research Centre of Russian Academy of Sciences, 112 Chernyshevsky str., 450008 Ufa, Russia

E-mail address: yurikor@matem.anrb.ru 